\title{
吡喃盐化合物的合成及应用研究进展
}

\author{
叶俊伟王萧㲏高远杨立健林源宁桂玲* \\ (大连理工大学化工与环境生命学部化工学院 精细化工国家重点实验室 大连 116024)
}

\begin{abstract}
摘要 吡喃盐是一类具有独特化学反应活性和光物理性质的杂环化合物，在有机合成、生物传感和光电材料等许多领 域展现了良好的应用前景. 结合本课题组的研究成果, 综述了 $\alpha$-非活性吡喃盐和 $\alpha$-活性吡喃盐化合物的合成研究进展, 并介绍了一些典型吡喃盐化合物在有机合成、光敏剂、离子液体和苂光传感材料等领域的应用.
\end{abstract}

关键词 吡喃盐; 合成; $\alpha$-活性; $\alpha$-非活性

\section{Research Progress on the Synthesis and Application of Pyrylium Salts}

\author{
Ye, Junwei Wang, Xiaoxiao Gao, Yuan Yang, Lijian Lin, Yuan Ning, Guiling* \\ (State Key Laboratory of Fine Chemicals and School of Chemical Engineering, Faculty of Chemical, Environmental and \\ Biological Science and Technology, Dalian University of Technology, Dalian 116024)
}

\begin{abstract}
Pyrylium salts are a type of heterocyclic compounds with unique chemical reactivity and photophysical properties. They show great potential applications in the many fields such as organic synthesis, biosensor and organic optoelectronic materials. Based on our recent research results, in this review, the developments in the synthesis of both $\alpha$-non-active pyrylium salts and $\alpha$-active pyrylium salts are summarized, and the applications of some typical pyrylium salts in the organic synthesis, photosensitizers, ion liquid and fluorescent sensor are exemplified.
\end{abstract}

Keywords pyrylium salts; synthesis; $\alpha$-active; $\alpha$-non-active

吡喃盐又称作吡喃鈆盐，是由吡喃盐阳离子和作为 抗衡离子的路易斯酸阴离子组成的六元氧杂环化合物. 常见的抗衡阴离子有 $\mathrm{Cl}^{-}, \mathrm{ClO}_{4}^{-}, \mathrm{SbCl}_{6}^{-}, \mathrm{BF}_{4}^{-}$, $\mathrm{FeCl}_{4}^{-}, \mathrm{AlCl}_{4}^{-}$等. 吡喃阳离子可被视为苯环上的一个 $\mathrm{CH}$ 被氧原子 $\left(\mathrm{O}^{+}\right)$取代的芳香体系, 但它的芳香性相比 苯环较差. 吡喃阳离子芳香环上的正电荷并不是平均分 布在六元杂环上, 而是主要分布在吡喃正离子芳香环的 邻位碳原子 $(\alpha-\mathrm{C})$ 和对位碳原子上 $(\gamma-\mathrm{C})$, 这样的电子结 构决定了它的独特光物理性质和化学反应活性, 使得它 在许多领域具有广泛的应用和重要价值 ${ }^{[1 \sim 3]}$. 作为一种 重要的有机合成中间体, 吡喃盐被广泛用于合成有机化 合物，其中报道最多的是吡喃盐与亲核试剂的反应 ${ }^{[4 \sim 6]}$, 常见的含氮、磷、硫亲核试剂均能与吡喃盐发生反应, 通 过环转化反应可以得到结构多样的有机环烃和杂环化 合物. 作为一种重要的功能分子, 吡喃盐化合物在染
料、激光材料、Q-开关材料、有机发光材料和非线性光 学材料等领域都得到广泛应用 ${ }^{[7 \sim 11]}$. 此外, 近年来, 该 类化合物在纳米器件、苂光传感器、金属有机化学、光 敏剂领域也展现了良好的应用前景 ${ }^{[12 \sim 14]}$, 吸引了人们 极大的研究兴趣.

吡喃盐化合物的性质在很大程度上取决于吡喃环 上取代基的类型和结构. 在报道的文献中 ${ }^{[1 \sim 6]}$, 大多数 吡喃盐化合物属于 $\alpha$-非活性吡喃盐 $(\alpha$ 位含有取代基), 其合成方法基本采用链状化合物经过多步缩合、取代或 加成, 最后环化得到 ${ }^{[1,15,16]}$. 仅有极少数 $\alpha$-活性吡喃盐 $(\alpha$ 位不含取代基或含一个取代基)被报道 ${ }^{[4]}$, 产物结构单 一，具有很大局限性. 本课题组在前期研究中发现，利 用苯基取代环戊二烯氧化扩环反应可以一步生成吡喃

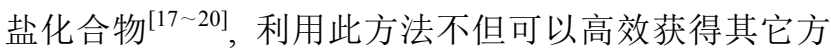
法难以合成的 $\alpha$-活性多苯基取代吡喃盐，并且该类 $\alpha$ 活

*E-mail: ninggl@dlut.edu.cn

Received September 10, 2014; revised October 11, 2014; published online October 21, 2014.

Project supported by the National Natural Science Foundation of China (Nos. 51003009, 20772014), the Fundamental Research Funds for the Central Universities of China (No. DUT14LK32) and the Science and Technology Research Foundation of Education Department of Liaoning Province (No. L2014033). 国家自然科学基金(Nos. 51003009, 20772014)、中央高校基本科研业务费(No. DUT14LK32)和辽宁省教育厅科学技术研究(No. L2014033)资助项目. 
性吡喃盐展现了独特的化学反应活性和光电性质 ${ }^{[21-25]}$. 本文按吡喃阳离子环上 $\alpha$ 位取代基种类进行分类，综述 了 $\alpha$-非活性吡喃盐和 $\alpha$-活性吡喃盐的合成进展, 其中包 括了吡喃酮法、1,5-戊二酮法、查尔酮和苯乙酮衍生物 反应法、缩二苯乙酮和酰氯反应反应法、炔酮合成法、 环戍二烯开环法等, 并对一些典型吡喃盐化合物在有机 合成、光敏剂、离子液体和苂光传感材料等领域的应用 作以介绍.

\section{$1 \alpha$-非活性吡喃盐的合成}

\section{1 吡喃酮法合成吡喃盐}

吡喃酮法合成吡喃盐是研究比较早的合成吡喃盐 的方法，比较典型的反应是吡喃酮与烷基化或酰基化试 剂进行亲电加成反应生成吡喃盐 ${ }^{[26]}$. 如 Eq. 1 所示, 利 用 4-吡喃酮衍生物为原料可以合成 4-甲氧基吡喃盐 1 . 利用 2-吡喃酩化合物为原料时, 需要利用更强的烷基化 试剂才能合成吡喃盐, 例如使用三烷基氧鎓氟硓酸盐. 4-吡喃酮与亲电试剂如 $\mathrm{PCl}_{5}, \mathrm{PBr}_{5}$, 或 $\mathrm{POCl}_{3}, \mathrm{COCl}_{2}$ 反 应可以生成 4-卤代吡喃盐 ${ }^{[27]}$.<smiles></smiles>

\section{2 饱和 1,5-戊二酮法合成吡喃盐}

饱和的 1,5-戊二酮化合物在酸催化下脱水生成不稳 定的吡喃环, 在氢负离子接收剂的作用下脱氢生成稳定 的吡喃盐 ${ }^{[15]}$. 最常见的合成 2,4,6-三取代吡喃盐的合成 方法见 Scheme 1. 芳醛和两分子的芳基烷基酮在路易斯 酸、强无机酸或脱水剂的存在下发生缩合反应生成饱和 1,5-戊二酮, 然后经环化脱氢生成 2,4,6-三取代吡喃盐 $\mathbf{2}^{[28]}$. 在这个过程中酸和脱水剂通常选用三氟化硼乙醚 络合物、高氯酸、氟硼酸或多聚磷酸等.

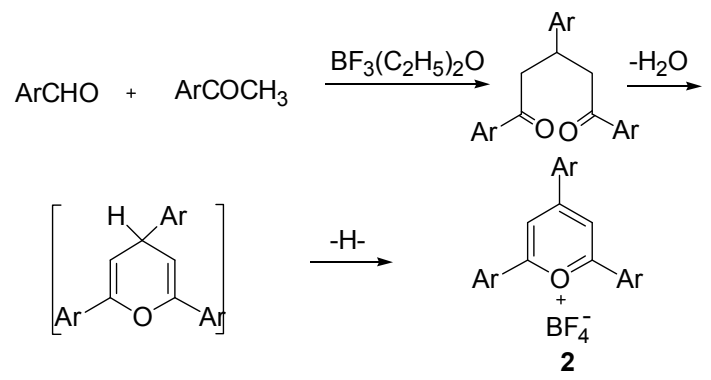

图式 1 饱和 1,5-戊二酮衍生物合成吡喃盐

Scheme 1 Synthesis of pyrylium salts from saturated penta-1,5dione derivates

根据 1,5-二酮类化合物结构不同, 可衍生出不同的

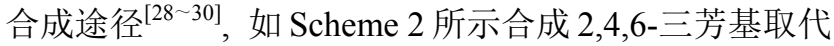
吡喃盐 3 的合成路线.

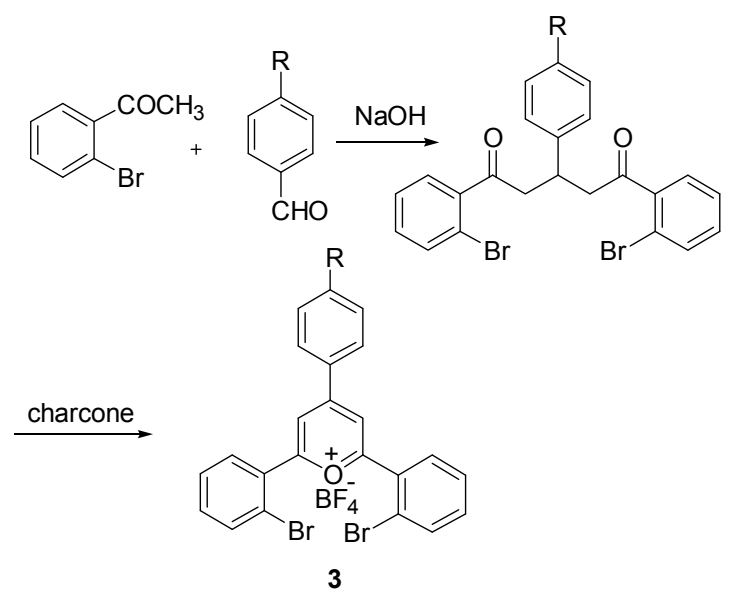

图式 2 饱和 1,5-戊二酮衍生物合成吡喃盐

Scheme 2 Synthesis of pyrylium salts from saturated penta-1,5dione derivates

\section{3 不饱和 1,5-戊二酮法合成吡喃盐}

不饱和 1,5-戊二酮类化合物在酸性环境中可以生成 吡喃盐. 如乙酰丙酮与苯乙酮反应可以生成 4,6-二甲基2-苯基高氯酸吡喃盐 4 (Scheme 3) ${ }^{[31]}$. 研究表明这个过 程是可逆的, 加入碱不利于吡喃盐的生成.

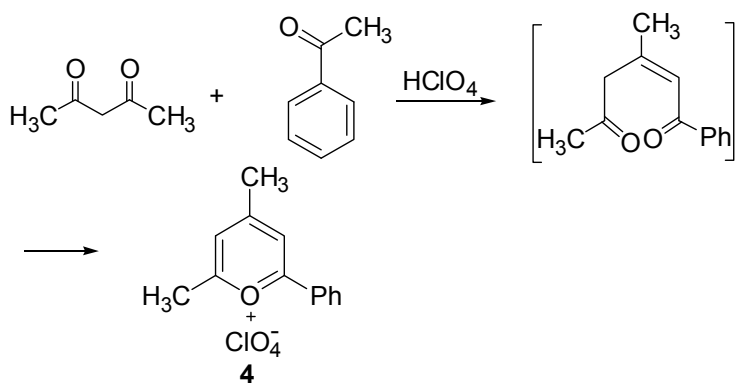

图式 3 4,6-二甲基-2-苯基高氯酸吡喃盐的合成 Scheme 3 Synthesis of 4,6-dimethyl-2-phenyl pyrylium perchlorate

烯烃和酰氯反应也能合成不饱和 1,5-戊二酮中间 体, 进而在酸性环境中合成吡喃盐, 该方法也称作 Balaban 合成法. 如 Scheme 4 所示, 异戊烯和乙酰氯反 应生成不饱和 1,5-戊二酮衍生物, 在酸作用下可以合成 2,3,4,6-四甲基吡喃盐 $\mathbf{5}^{[32]}$. 反应过程一般认为是烯烃酰 化生成碳正离子中间体，然后脱氢生成烯醇后脱水生成 目标产物.

丙酮缩合可以得到异丙叉丙酮, 在酸性条件下生成 不饱和 1,5-戊二酮化合物, 之后环化也可以得到 2,4,6三甲基高氯酸吡喃盐 6 (Scheme 5) ${ }^{[33]}$. 
<smiles></smiles>

图式 4 2,3,4,6-四甲基高氯酸吡喃盐的合成 Scheme 4 Synthesis of 2,3,4,6-tetramethyl pyrylium perchlorate<smiles>CC(=O)C=CC(C)=CC(C)=O</smiles><smiles>CC(=O)C=C(C)CC(C)=O</smiles>

图式 5 2,4,6-三甲基高氯酸吡喃盐的合成 Scheme 5 Synthesis of 2,4,6-trimethyl pyrylium perchlorate

\section{4 查尔酮和苯乙酮衍生物反应法合成吡喃盐}

利用查尔酮和苯乙酮衍生物在路易斯酸的存在下 反应可以获得 2,3,4,6-四取代吡喃盐 7 (Eq. 2) ${ }^{[16]}$.<smiles>O=C(/C=C/c1ccccc1)c1ccccc1</smiles><smiles>[R]CC(=O)c1ccccc1</smiles>

7

\section{5 缩二苯乙酮和酰氯反应法合成吡喃盐}

Hopf 等 ${ }^{[34}$ 报道了利用缩二苯乙酮和酰氯反应合成 吡喃盐的方法(Eq. 3), 之后, Katritkzky 等 ${ }^{[35,36]}$ 对此方法 进行了优化改进并被广泛应用于合成简单的手性吡喃 盐化合物.

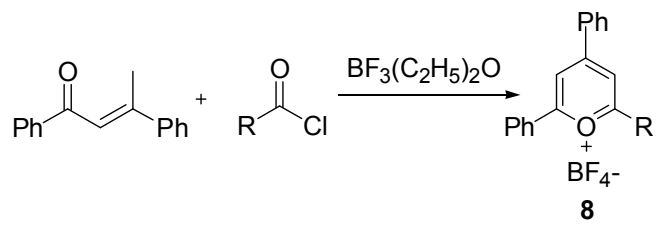

\section{6 炔酮法合成吡喃盐}

炔酮合成法合成吡喃盐和 1,5-二酮法相似，不同的 是在 1,5-二酮合成方法中是两个羰基缩合, 而在炔酮合 成法中是一个羰基和一个炔基的缩合 ${ }^{[37,38]}$. 如 Scheme 6 所示, 利用炔酮合成法可以合成 1-苯并吡喃盐类化合物 9.

需要关注的是, 苯并吡喃盐类化合物作为重要的天 然产物近年来引起人们极大关注, 例如 3,5,7-三差基-

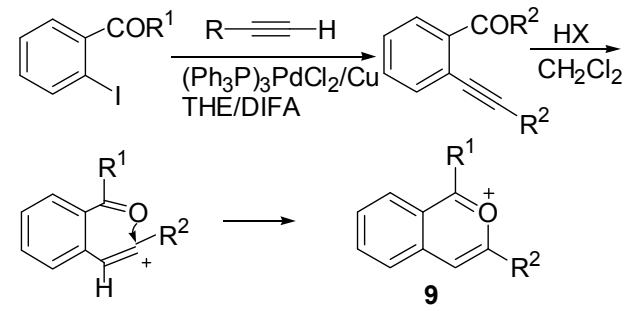

图式 6 炔酮缩合法合成吡喃盐

Scheme 6 Synthesis of pyrylium salts by condensation reaction between carbonyl and alkynyl groups

2-(3,4-二羟基苯基)-1-苯并吡喃盐(花青素氯化物). 但其 合成方法仍局限于利用酚醛缩合或者醛酮缩合方法. 例 如，利用邻羟基苯乙酮类化合物与原酸酯和芳醛发生环 缩合反应可以生成 4-烷氧基-2-芳基-1-苯并吡喃盐; 再 例如，利用酸性介质条件下，邻着基苯甲醛或苯乙酮与 亚甲基酮发生缩合反应可以合成 1-苯并吡喃盐(Scheme $7)^{[39,40]}$. 本文对该类化合物的合成作不做更多的描述.
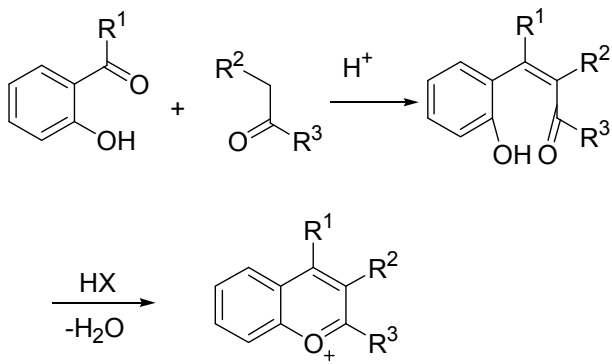

$10^{X^{-}}$

图式 7 醛酮缩合法合成吡喃盐

Scheme 7 Synthesis of pyrylium salts by condensation reaction between carbonyl aldehyde groups

\section{7 环戊二烯开环法合成吡喃盐}

2004 年, 我们课题组 ${ }^{[17]}$ 首次报道了高氯酸银存在 条件下实现了氧原子插入环戌二烯环合成 2,3,4,5,6-五 苯基高氯酸吡喃盐的方法，该方法中 $\mathrm{Ag}^{+}$既起到了路易 斯酸催化剂作用，也是氧化剂促进了氧原子的插入和环 戊二烯环的开环。最近，我们课题组 ${ }^{[18]}$ 又利用 $\mathrm{FeCl}_{3} \cdot 6 \mathrm{H}_{2} \mathrm{O}$ 催化氧化多芳基取代环戍二烯一步法合成 了系列 $\alpha$-非活性吡喃盐化合物，利用单晶衍射分析证实 了产物结构为 2,4,6-三芳基取代四氯合铁吡喃盐 11 和 2,3,4,6-四芳基取代四氯合铁吡喃盐 12 (Eq. 4). 该方法 具有反应条件温和、产率高、选择性强特点, 不需要严 格的无水无氧条件也可以获得产物，更为重要的是合成 的产物四氯合铁吡喃盐化合物具有良好的水稳定性和 强的荧光发射.

\section{8 吡喃盐的其他合成方法}

近年来，关于吡喃盐合成方法的改进和新制备路线 的开发不断被报道. 例如，利用取代的苯甲酸或苯甲酸 

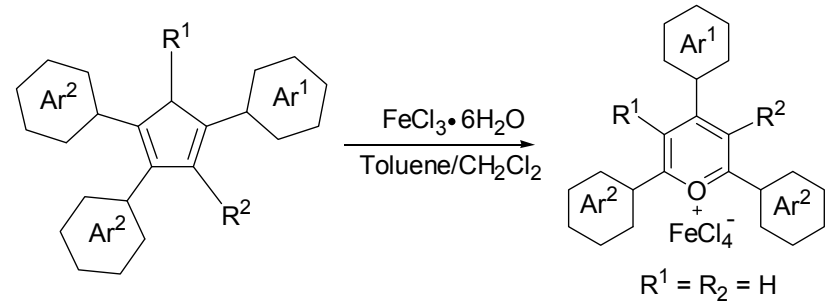

11

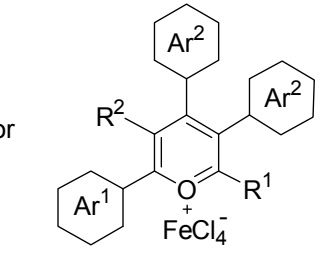

$\mathrm{R}^{1}=\mathrm{Ph}, \mathrm{R}^{2}=\mathrm{H}$

12<smiles></smiles><smiles></smiles>

$11 \mathrm{a}$

11c<smiles></smiles>

11b

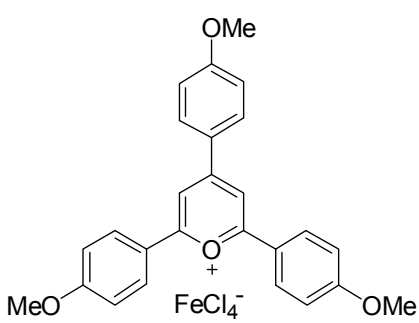

11d
甲酯和取代的苯乙酮反应合成 2,4,6-三芳基取代的四氟 化嗍吡喃盐 13 (Eq. 5) ${ }^{[41]}$.<smiles>[R]C(=O)c1ccc([R])cc1</smiles><smiles></smiles>

13

利用酯基丙炔、二苯乙炔和过渡金属配合物
$\mathrm{Mn}(\mathrm{CO})_{5} \mathrm{BF}_{4}$ 反应(Eq. 6), 得到一种金属-吡喃盐配合物 结构 $14^{[38]}$.

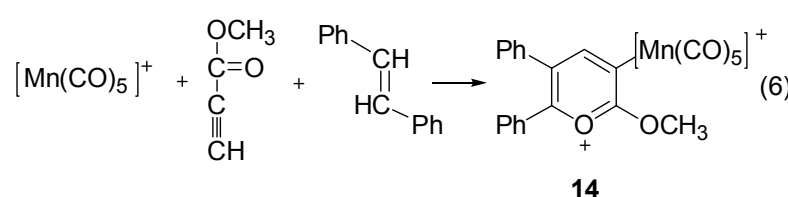

邻位被锰取代的查尔酮与乙炔基二茂铁在四氯化 碳中回流反应(Scheme 8), 生成 2 位有二茂铁基取代的 吡喃盐化合物 $\mathbf{1 5}^{[42]}$.<smiles>O=C(/C=C/c1ccccc1)c1ccccc1</smiles>

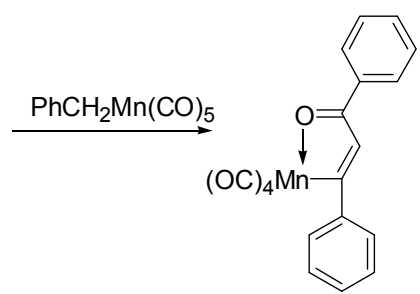

$\underset{\mathrm{FcCCH} / \mathrm{CCl}_{4}}{\longrightarrow}$<smiles>[X]c1c(-c2ccccc2)cc(-c2ccccc2)cc1-c1cccc(Oc2ccccc2)c1</smiles>

图式 8 二茂铁取代吡喃盐的合成

Scheme 8 Synthesis of ferrocene-substituted pyrylium salt

不饱和的腈类化合物经高氯酸和醋酸混酸作用也 可进行一步缩合反应(Eq. 7)生成吡喃盐 $16^{[43]}$.

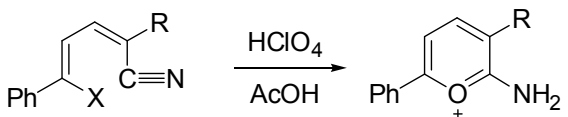

$$
\begin{aligned}
& \mathrm{X}=\mathrm{OH} \quad \mathrm{R}=\mathrm{CO}_{2} \mathrm{Et}, \mathrm{CONH}_{2}{ }^{16}
\end{aligned}
$$

$\beta$-氯代不饱和羰基化合物与甲基或亚甲基酮在高 氯酸和醋酸混酸作用下也可以缩合(Eq. 8)生成 2,5-二取 代吡喃盐 $17^{[44]}$.
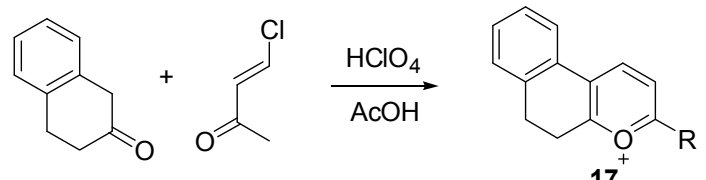

\section{$2 \alpha$-活性吡喃盐的合成}

\section{1 吡啶合成 $\alpha$-活性吡喃盐}

$\alpha$-活性吡喃盐的合成比较困难, 相关的研究很少, 
不含取代基的 $\alpha$-活性吡喃盐 18 (Scheme 9) 比较早被人 们认识 ${ }^{[4]}$. 然而这种方法合成的吡喃盐结构比较单一, 限制了其应用.

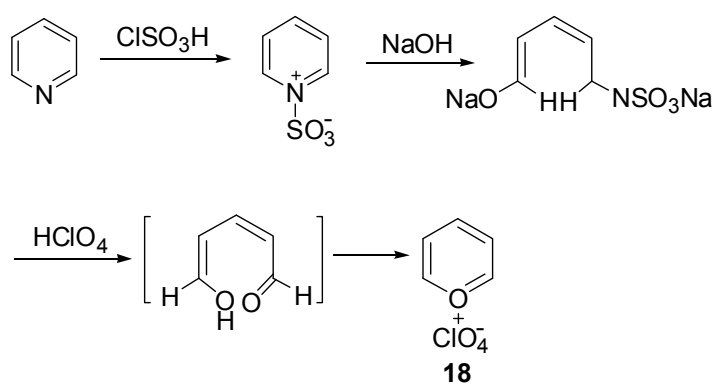

图式 9 吡啶合成无取代基吡喃盐

Scheme 9 Synthesis of unsubstituted pyrylium salt from pyridine

\section{2 环戊二烯开环法合成 $\alpha$-活性吡喃盐}

2004 年, 我们课题组 ${ }^{[17]}$ 首次报道了苯基取代的环 戊二烯化合物在高氯酸银存在条件下可以发生氧原子 插入环戊二烯环反应(Eq. 9), 并首次合成了 $\alpha$-活性 2,4,5-四苯基高氯酸吡喃盐 19 和银配位 2,3,4,5-四苯基高 氯酸吡喃盐 20.2005 年, 我们课题组 ${ }^{[19]}$ 证实了酸性条件 下氧原子也可以掺入环戊二烯环获得吡喃盐化合物. 2013 年, 我们 ${ }^{[20]}$ 利用修饰后的三芳基取代环戍二烯作 为原料, 采用酸/空气氧化方法获得系列新的 $\alpha$-活性吡 喃盐 21 (Eq. 10), 通过 X 射线单晶结构分析和量子化学 计算方法对它们的结构进行了研究. 此外这样的简便合 成方法可以通过调变取代基团结构获得绿光到红光发 射的多色彩吡喃盐化合物.

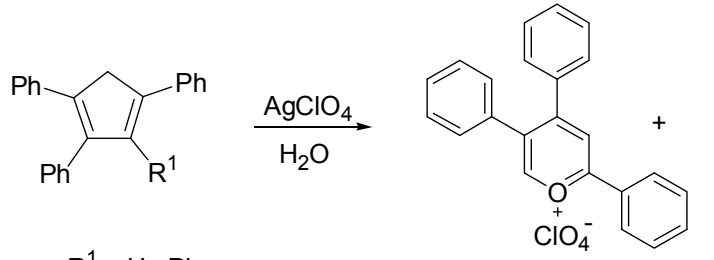

$\mathrm{R}^{1}=\mathrm{H}, \mathrm{Ph}$

19<smiles>Oc1cc2c3ccccc3c1=C(c1ccccc1)C(c1ccccc1)=C(c1ccccc1)C=2c1ccccc1</smiles>

20

我们课题组 ${ }^{[18]}$ 最近研究发现利用 $\mathrm{FeCl}_{3} \cdot 6 \mathrm{H}_{2} \mathrm{O}$ 代替 高氯酸银也可以合成 $\alpha$-活性吡喃盐. 常温条件下可溶性 铁金属离子促进芳基取代环戊二烯的氧化扩环反应是
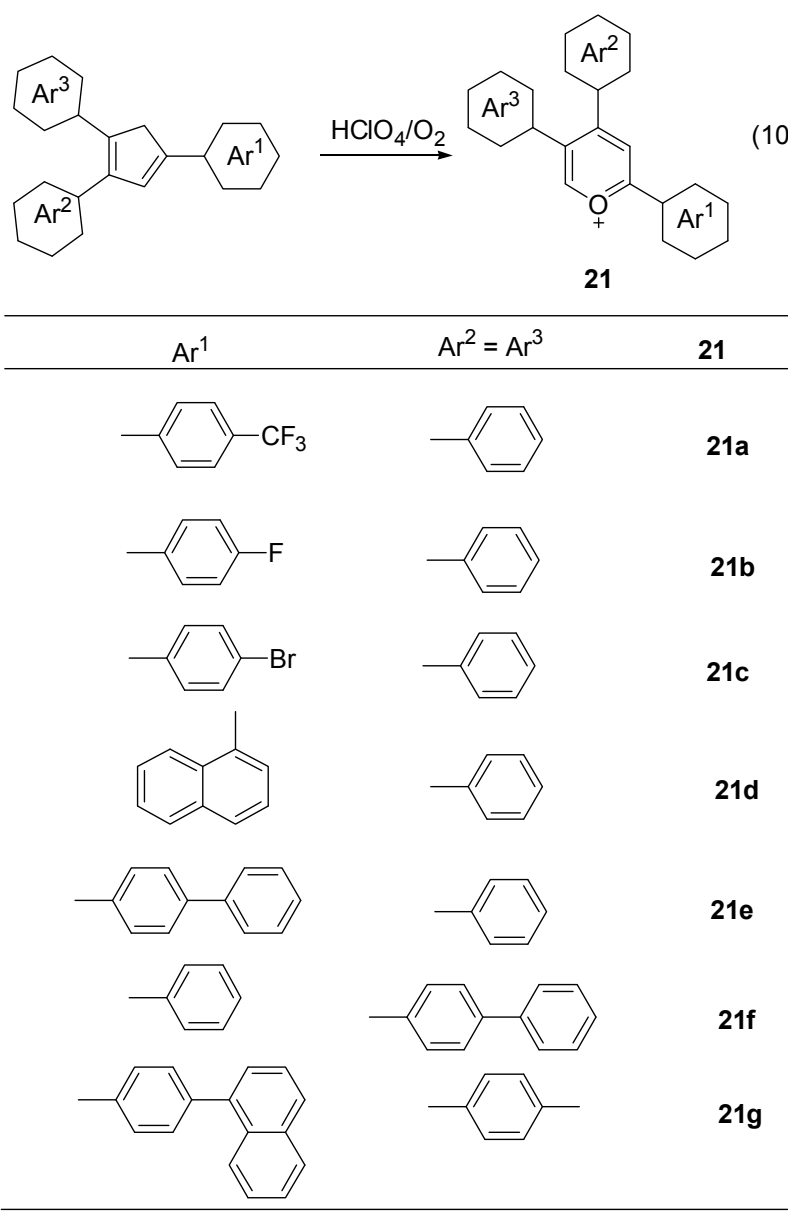

一种此前未见文献报道的吡喃盐合成方法. 对比之下, 环戊二烯开环法具有反应条件温和、一步合成、产率高 的特点, 不仅可以合成出传统的吡喃盐, 而且还可以非 常简便地合成传统方法难合成的 $\alpha$-活性吡喃盐, 极大开 拓了吡喃盐化合物的合成途径.

\section{3 其他方法合成 $\alpha$-活性吡喃盐}

文献中也有一些其他反应合成 $\alpha$-活性吡喃盐. 例 如, 利用苯乙酮和 1,1,3,3-四乙氧基丙烷反应合成 $\alpha$ 单取 代吡喃盐(Eq. 11) ${ }^{[45]}$, 利用苯乙酮和肉桂醛反应合成 2,4-二芳基取代的吡喃盐(Eq. 12) ${ }^{[46]}$.

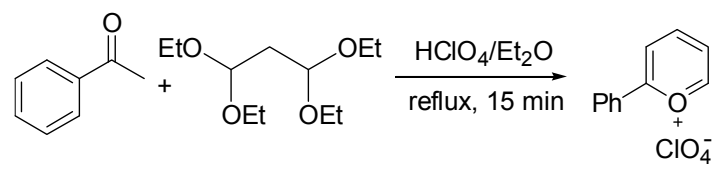

22

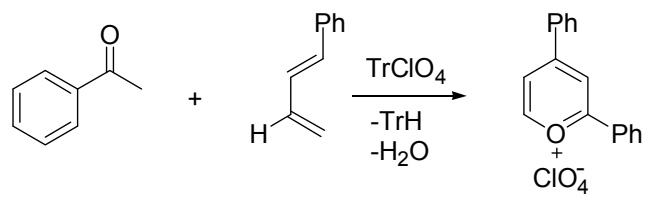


综上所述, 关于吡喃盐的合成研究发展迅速, 合成 方法不断得到改进. 然而, 合成过程仍然主要从链状化 合物出发经过多步环化反应实现, 步骤较多, 产率较低, 而且受此方法所限, 很难合成 $\alpha$-活性吡喃盐化合物. 报 道的绝大多数吡喃盐都是 $\alpha$-非活性的 2,4,6-三取代吡喃 盐、2,3,4,6-四取代吡喃盐或者是 2,3,4,5,6-五取代 $\alpha$-非活 性吡喃盐化合物, $\alpha$-活性吡喃盐很少报道. 开发新的吡 喃盐合成路径, 特别是合成 $\alpha$ 单取代吡喃盐化合物对丰 富吡喃盐的种类, 开拓吡喃盐在有机合成中的应用, 合 成新型化合物具有重要意义.

\section{3 吡喃盐的应用}

吡喃盐阳离子存在四种共振结构, 如 Scheme 10 所 示, 正电荷主要分布在邻位碳原子 $(\alpha-\mathrm{C})$ 和对位碳原子 $(\gamma-\mathrm{C})$ 上, 这样的电子结构决定了其独特的光物理性质 和化学反应活性 ${ }^{[1]}$, 使其在很多领域具有广泛的应用, 这里对一些典型吡喃盐化合物在有机合成、光电材料、 离子液体等领域的应用作以介绍.

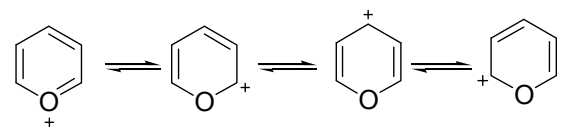

图式 10 吡喃盐阳离子的电荷分布

Scheme 10 Charge distribution of pyrylium cation

\section{1 吡喃盐在有机合成中的应用}

由于吡喃盐特殊的共振结构, 它非常容易和亲核试 剂发生反应, 反应一般发生在邻位碳原子 $(\alpha-C)$ 和对位 碳原子上 $(\gamma-\mathrm{C})$. 虽然理论计算的结果证明 $\alpha$ 位和 $\gamma$ 位上 正电荷分布相似, 但是由于氧原子的强诱导效应， $\alpha$ 位 上更缺电子, 所以相对于 $\gamma$ 位, 亲核反应更容易在 $\alpha$ 位 发生, 亲核试剂进攻 $\alpha$ 位生成不稳定的 $\alpha$-吡喃环, 之后 发生开环进行后续反应 ${ }^{[1]}$. 吡喃盐能和多种亲核试剂反 应, 如含杂原子 $\mathrm{N}, \mathrm{P}, \mathrm{O}, \mathrm{S}$ 的有机亲核试剂, 产物的结构 多种多样, 包括环状烃类化合物、杂环化合物和链状化 合物, 因此吡喃盐是有机合成领域非常重要的原料或中 间体.

\subsection{1 吡喃盐与含 $\mathrm{N}$ 试剂的反应}

吡喃盐可以与含氮有机亲核试剂(如氨气、羟基胺、 芳基胺等)反应生成含氮杂环化合物 ${ }^{[4]}$. 由吡喃盐转化 成吡啶环的反应条件温和, 操作简单, 产物分离容易, 是制备吡啶化合物非常简单高效的途径. 吡喃环上取代 基的数量对反应产率有直接影响, 一般来说, 取代基的 数量越多, 吡喃环化为吡啶环的产率越高. 近年来, 吡 喃盐与伯胺反应生成吡啶阳离子被应用于构建具有特
殊分子结构和性质的吡啶盐阳离子化合物 ${ }^{[47 ~ 50]}$. 例如 利用吡喃盐与氨基吡啶的反应(Eq. 13), 得到具有光敏 超分子自组装性质的三齿吡啶类化合物 ${ }^{[7]}$.
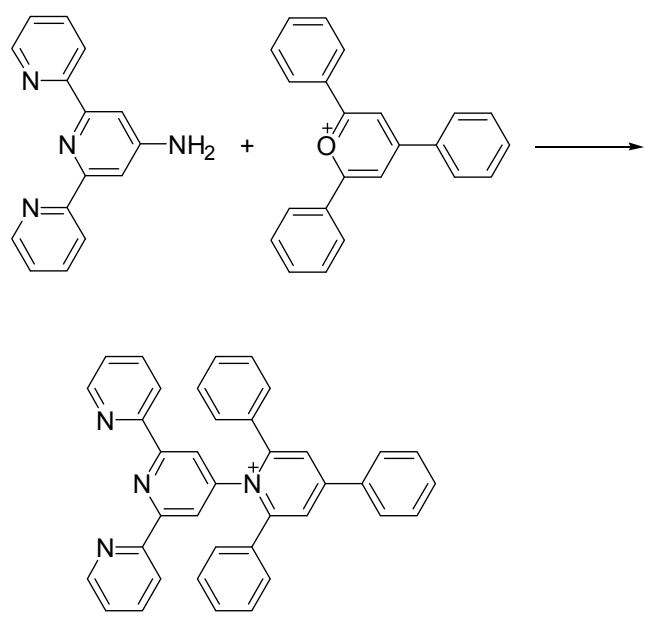

利用双吡喃盐 24 和二胺反应, 能够得到新颖的聚 吡啶盐结构(Eq. 14) ${ }^{[48]}$. 该聚吡啶盐具有很大的抗衡阴 离子(对甲苯磺酸根), 在质子型和非质子型有机溶剂中 表现出溶致液晶的性质. 另外这类化合物容易成膜，并 且具有很好的光致发光性能, 使其在聚合物发光器件有 潜在的应用。

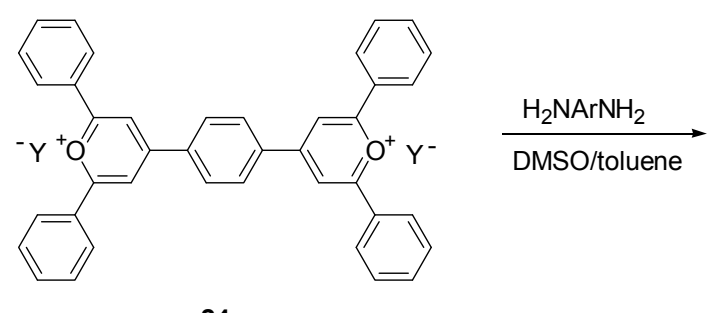

24

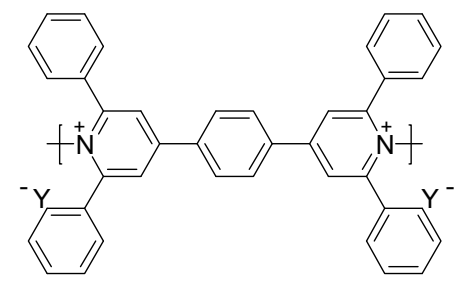

我们课题组 ${ }^{[25]}$ 利用 $\alpha$-活性吡喃盐为原料和有机胺 反应合成了系列吡啶盐化合物, 这类吡啶盐易于发生分 子内的环化反应合成发光的苯并咪唑类衍生物(Scheme 11). 同时利用该类吡啶盐的光化学反应也可以制备出 具有共平面结构的菲啶盐化合物, 并且发现吡啶盐 $\mathrm{N}$ 取 代基上强供电基团的引入不利于吡啶盐发生光环化反 应，而通过取代基改变可以获得具有不同发光颜色的苯 并咪唑类衍生物. 
<smiles>[R]c1cc(CC)ccc1N</smiles>

19

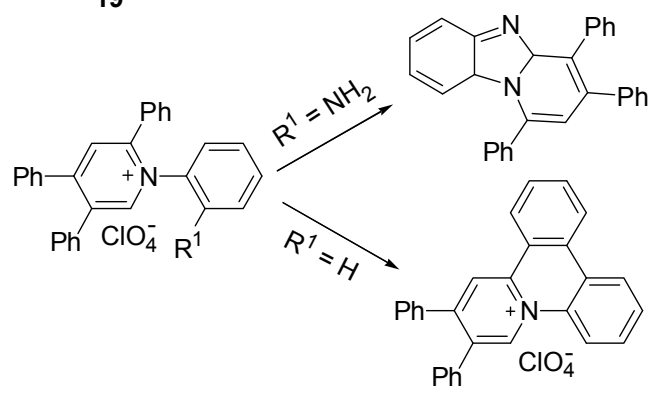

图式 11 苯并咪唑衍生物和菲啶高氯酸盐的合成 Scheme 11 Synthesis of benzimidazole derivates and phenanthridine perchlorate

Müller 等 ${ }^{[49]}$ 也利用吡喃盐为原料, 通过胺化和光合 环反应合成了系列两亲分子稠环芳烃化合物 (Scheme 12), 并组装了一维纳米纤维、纳米带和管状结构.

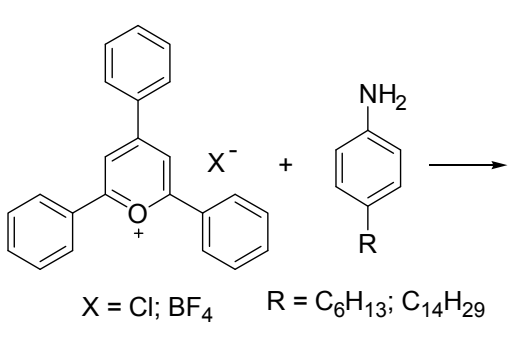

11a

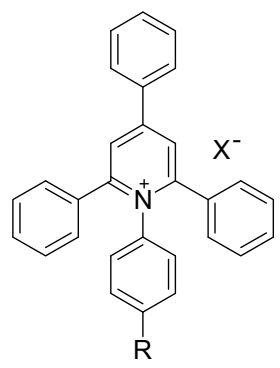<smiles>[R]c1cc2c3ccccc3c3cc(-c4ccccc4)cc(-c4ccccc4-c4ccccc4)n3c-2c1</smiles>

图式 12 菲啶盐衍生物的合成

Scheme 12 Synthesis of phenanthridinylium salt derivates

吡喃盐还可以与差胺反应(Eq. 15), 进而得到吡啶1-氧化物和 2-异啞唑啉 ${ }^{[50]}$. 其中合成的异啞唑啉是一类 应用广泛的药效基团, 经常作为合成医药和复杂天然化 合物的中间体, 如 GPII/IIIa 抑制剂和人类白细胞弹性蛋 白酶抑制剂的合成. 用着弪胺盐酸盐代替着胺与吡喃盐反 应，采用无溶剂的微波合成法，反应产物为单一的 2-异 啞唑啉, 实现了利用吡喃盐高效率、选择性合成 2-异噁 唑啉 ${ }^{[51]}$.<smiles>[X][X]C([R])=CC1([R])CC([R])=NO1</smiles>

\subsection{2 吡喃盐与含 $\mathrm{P}$ 试剂的反应}

近年来, 对 2,4,6-三苯基吡喃盐、2,6-二苯基吡喃盐 与三配位有机膦试剂的反应已有很多的研究. 目前利用 有机膦试剂与 2,4,6-三苯基吡喃盐反应已成为合成膦杂 苯的一条重要途径, 并且这种制备方法还可以得到传统 方法得不到的膦杂苯配体. 膦杂苯化合物是有机金属配 位化学领域中一类重要的配体. 利用三羟甲基膦和三硅 甲基膦分别进攻吡喃盐(Scheme 13), 都能够得到膦杂 苯化合物，而后者的产率比前者的更高 ${ }^{[5,52,53]}$. 得到的 二膦杂苯配体结构新颖，可以与金属铑配位后生成配合 物，具有特殊的反应活性和催化性能 ${ }^{[14]}$.<smiles></smiles>

25

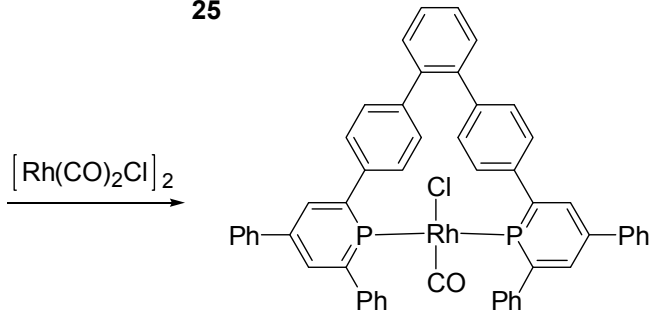

图式 13 有机膦配体的合成

Scheme 13 Synthesis of organophosphorus ligand

2,6-二苯基吡喃盐与三苯基膦反应可以得到二聚的 吡喃酮化合物，与三乙氧二乙基亚磷酸钠反应则可以生 成 $\gamma$-吡喃膦酸盐(Scheme 14) ${ }^{[54]}$.

我们课题组 ${ }^{[21]}$ 对 $\alpha$ 单取代 2,4,5-三苯基吡喃盐与三 配位有机膦试剂的反应进行了研究, 填补了 $\alpha$ 活性吡喃 盐与有机膦亲核试剂研究领域的空白. 选用四种有机膦 化合物与 2,4,5-三苯基吡喃盐反应(Scheme 15), 得到的 产物结构和之前报道的完全不同，既没有膦杂苯的产 生, 也没有二聚吡喃酮的产生. 产物结构与原料配比有 密切关系, 当两种原料按 $1: 1$ 的配比反应时, 得到的产 物为开环膦盐结构; 当有机膦试剂过量时, 得到产物为 环戊二烯膦盐结构. 说明 $\alpha$-活性吡喃盐具有不同于 $\alpha$-非 
活性吡喃盐的独特反应活性, 在有机合成领域具有重要 意义.

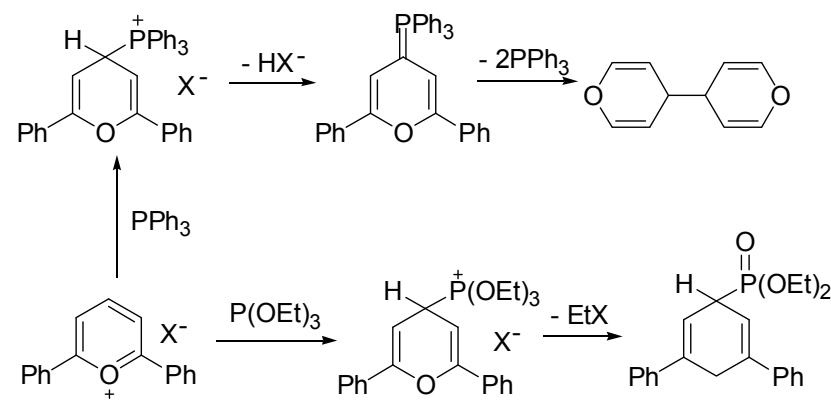

26

$(\mathrm{EtO})_{2} \mathrm{PONa}$

$-\mathrm{NaX}$

图式 14 2,6-二苯基吡喃盐与有机膦的反应

Scheme 14 Reaction of 2,6-biphenyl pyrylium salt with organophosphorus reagents<smiles>CC(C)(C)C1(c2ccccc2)COC(c2ccccc2)=CC1=Pc1ccccc1</smiles>

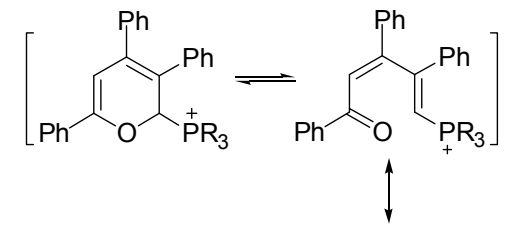

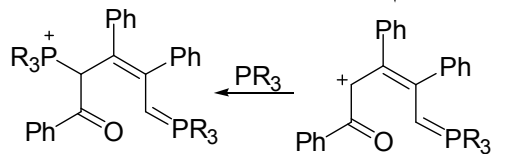<smiles></smiles>

图式 $152,4,5$-三苯基吡喃盐与有机膦的反应

Scheme 15 Reaction of 2,4,5-triphenylpyrylium salt with organophosphorus reagents

\subsection{3 吡喃盐与含 $\mathrm{S}$ 试剂的反应}

吡喃盐与含硫亲核试剂反应可以生成噻喃盐. 相对 于吡喃盐, 噻喃盐具有更高的稳定性, 受环境的 $\mathrm{pH}$ 影 响较小, 通常在光催化反应中作为光敏剂使用, 也是一 类稳定的有机盐发光材料. 例如, 在吡喃盐的丙酮溶液 中加入硫化钠水溶液, 搅拌一定时间后加高氯酸酸化生 成噻喃盐(Scheme 16), 噻喃盐以大环为接收基团, 噻喃 环部分作为响应基团, 可选择性识别水溶液中的 $\mathrm{Hg}^{2+}$ 和 $\mathrm{Cu}^{2+}{ }^{[6]}$.

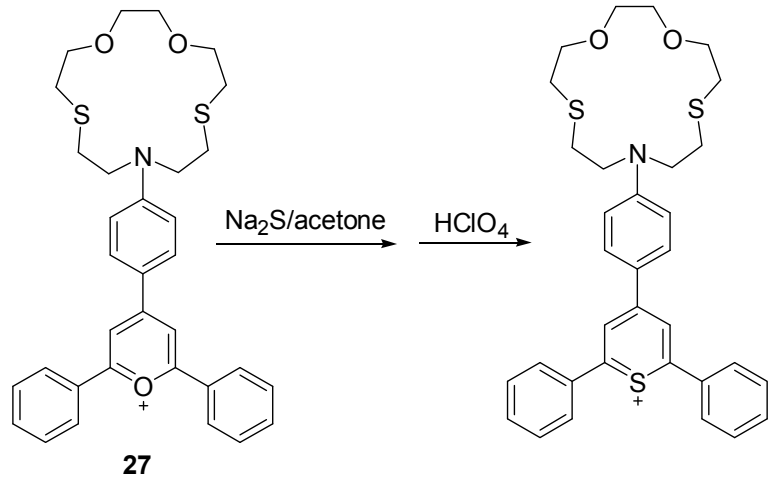

图式 16 吡喃盐与硫亲核试剂的反应

Scheme 16 Reaction of pyrylium salt with sulfur nucleophilic reagent

\section{1 .4 吡喃盐与含 $\mathrm{O}$ 亲核试剂的反应}

最简单的含氧亲核试剂是水和碱，吡喃盐水解的难 易程度与吡喃环上的取代基数目有重要关系, 不带取代 基的吡喃盐稳定性低. 报道的吡喃盐大都可以在碱的水 溶液中水解开环生成不饱和醇酮或不饱和 1,5-二酮 (Scheme 17), 可被视为合成吡喃盐的逆反应. 如果吡喃 环 $\alpha$-位连接甲基或亚甲基，水解得到的甲基酮或亚甲基 酮发生羟醛缩合, 生成苯衍生物 ${ }^{[55]}$.

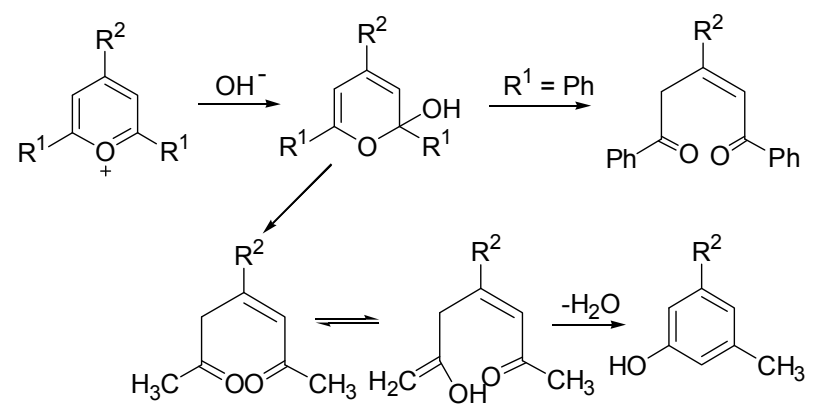

图式 17 吡喃盐与氧亲核试剂的反应

Scheme 17 Reaction of pyrylium salt with oxygen nucleophilic reagent

\subsection{5 吡喃盐开环合成癸四烯二酮化合物}

以吡喃盐为原料合成含有三个双键以上的共轭多 烯链状结构的报道很少, 研究领域处于起步阶段. 共轭 多烯化合物因其广泛的应用，关于其合成方法的探索一 直是近年来的研究热点. 传统的合成多烯类化合物一般 利用 Wittig-HWE 缩合反应或贵金属(如 Pd, Pt)催化交叉 偶联反应，然而这些方法通常步骤繁琐、产物的立体选 择性较差、产物分离纯化困难.

我们课题组 ${ }^{[23]}$ 以吡喃盐为原料, 金属为还原剂, 合 成了系列癸四烯二酮化合物(Scheme 18). 并且发现以 $\alpha$-活性吡喃盐为原料, 产物均为 $(2 Z, 4 Z, 6 Z, 8 Z)$ - 癸四烯二 酮化合物; 而以非 $\alpha$ 活性吡喃盐为原料, 产物结构具有 


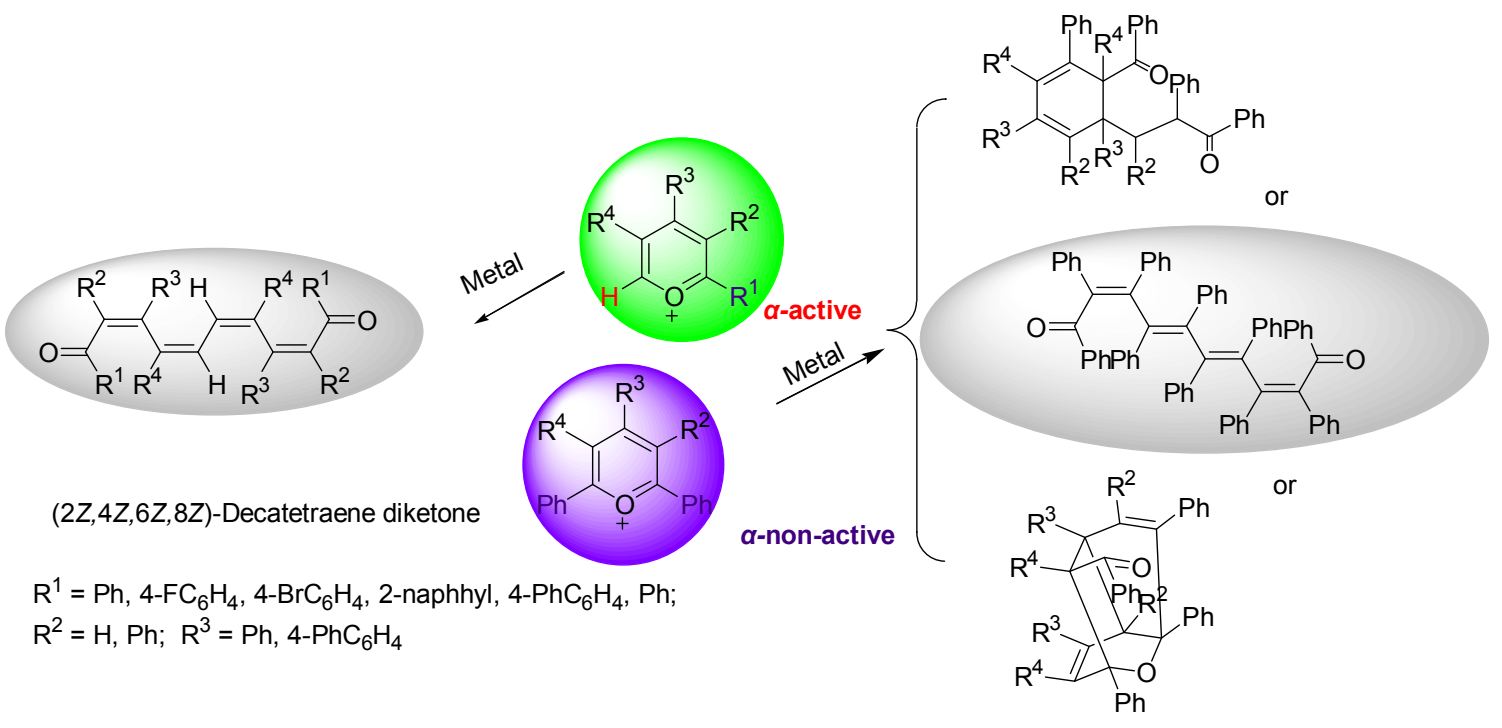

图式 18 吡喃盐合成多烯酮衍生物

Scheme 18 Synthesis of polyene diketone derivatives from pyrylium salts

多样性. 其中 $(2 Z, 4 Z, 6 Z, 8 Z)$ - 癸四烯二酮化合物具有较高 的热稳定性, 晶体分子结构呈现出扭转 ' $\mathrm{S}$ ' 形构型, 导 致其较低的苂光发射强度 ${ }^{[24]}$.

\section{2 吡喃盐作为光敏化剂}

处于光激发态的吡喃盐是良好的氧化剂, 在光化学 反应过程中, 不产生任何单线态氧或超氧化自由基, 这 极大程度减少了副反应的发生, 简化了光反应过程, 因 此可以作为一种高效的光敏化剂 ${ }^{[56]}$. 选择吡喃盐作为 带正电荷的电子受体(A), 与一个不带电荷的中性电子 给体(D)组成一个 $\mathrm{D} / \mathrm{A}$ 对, 在电荷转移之前或者之后, 电子给受体之间不会存在库伦引力, 从而减小了电子回 传(BET)过程, 可以提高光子能量的储存效率. 2,4,6-三 芳基吡喃盐很容易被可见光激发, 其良好的光敏化性能 已被广泛应用于光诱导电子转移 (PET) 过程. 其中, $2,4,6$-三苯基四氟化嗍盐 $\left(\mathrm{TPPBF}_{4}\right.$ ) 备受关注, 无论是在 单线态或激发三线态都是优良的氧化剂 ${ }^{[56]} . \mathrm{TPPBF}_{4}$ 光 敏化 PET 反应有很多种, 因为 $\mathrm{TPP}^{+}$可通过 $\mathrm{TPP}^{\circ}$ 与 $\mathrm{O}_{2}$ 的反应再生, 所以光敏化反应过程通常可在空气中或者 含饱和 $\mathrm{O}_{2}$ 的溶液中进行, 反应中只需加入催化剂量的 吡喃盐敏化剂. 如 Eq. 16 中所示, 在光辐射的条件下, $\mathrm{TPPBF}_{4}$ 在乙腈中敏化狮衍生物可以得到反立体选择性 的 head-to-head 二聚产物, 产率可以达到 $97 \%{ }^{[56]}$.
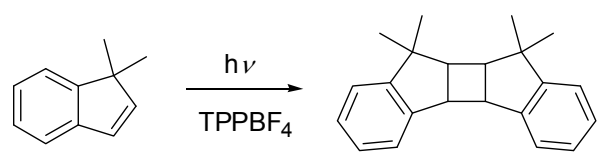

在乙腈或二氯甲烷溶液中, $\mathrm{TPPBF}_{4}$ 光敏化茮醇生 成芳香自由基中间体, 接着去质子化可以生成相应的苯
甲醛(Scheme 35$)^{[57]}$. Hurst 等 ${ }^{[58]}$ 最近也证实了 2,4-二苯 基-6-二甲胺基苯基吡喃盐作为光敏化剂和氧化还原介 质作用.

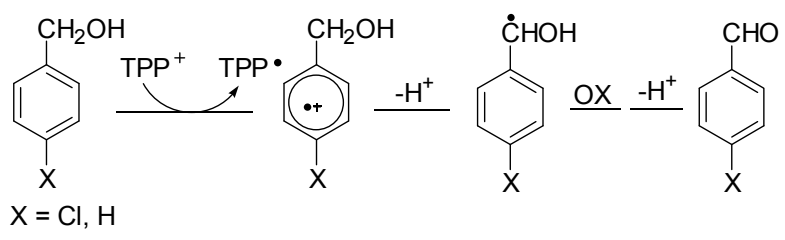

图式 $19 \mathrm{TPPBF}_{4}$ 敏化 PET 反应: 醇氧化

Scheme $19 \mathrm{TPPBF}_{4}$-sensitized PET reaction: oxidation of benzyl alcohol

\section{3 吡喃盐作为离子液体}

根据阳离子的不同，离子液体可分为以下几类：咪 唑盐、铵盐、吡啶盐、磷盐、哌啶盐、吗啉盐和硫盐离 子液体，它们的正电荷分别分布在氮、磷或硫原子上 ${ }^{[59]}$. 吡喃盐代表了一类正电荷位于氧原子上的离子液体. 2,6-二取代吡喃高氯酸盐作为离子液体被大量研究, 当 吡喃盐的 2 位和 6 位的苯基上连有长烷基链或者烷氧基 链时, 就可能具有离子液晶性质 ${ }^{[60,61]}$. 类似的, 当 2,4,6三苯基四氟化嗍吡喃盐的三个苯基的间位和对位被烷 氧基取代后，也具有离子液晶性质 ${ }^{[62]}$.

2011 年, Pernak 等 ${ }^{[63]}$ 报道了一类含有机抗衡阴离子 (磺酸根)的吡喃盐离子液体的合成方法(Eq. 17), 并且这 类离子液体很好地应用在氢化硅烷基化反应中.

\section{4 吡喃盐苂光传感材料}

吡喃盐化合物大多具有良好的荧光发射, 在有机发 


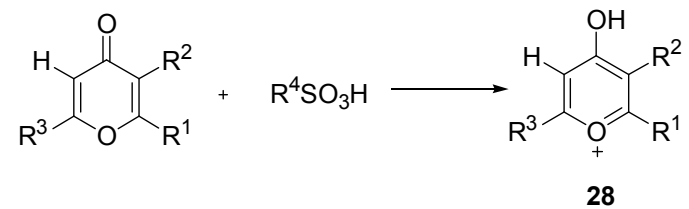

28

光、激光染料和光化学反应敏化剂方面有着广阔的应用 前景. 吴世康课题组 ${ }^{[64]}$ 对吡喃环上取代基的空间结构 或构象对吡喃盐的发光性能关系做了很多研究工作. Haucke 等 ${ }^{[65]}$ 对芳环上连有不同电子效应的取代基(即吸 电子和供电子取代基)时对吡喃盐化合物发光行为的影 响进行了分析. 利用吡喃盐与亲核试剂的反应, 可以开 发出反应型吡喃盐苂光探针材料. 通过 Huisgen-click 反 应, 将吡喃盐衍生物连接到 $\mathrm{mCP}$ 表面, 可以制备成微米 或纳米级反应平台, 利用吡喃盐与伯胺的反应特性检测 氨基化合物 ${ }^{[12]}$. Duerkop 等 ${ }^{[66]}$ 也报道了用于环境中脂肪 胺、芳香胺检测的吡喃盐化合物, 主要也是利用吡喃盐 和有机胺反应生成发光颜色不同的吡啶盐化合物来实 现的(Eq. 18).

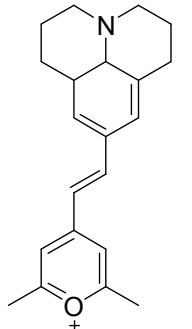

29

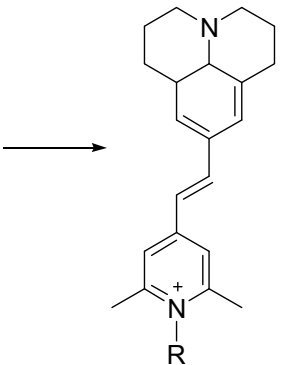

$\mathrm{R}$
吡喃盐和吡喃酮之间可以发生可逆转化, 两种化合 物的苂光性能差异较大. 利用吡喃盐与吡喃酮之间的转 化反应(Eq. 19), 可以构造基于氢键的质子传感器. 吡喃 酮化合物在加入酸后 $\left(\mathrm{CH}_{3} \mathrm{COOH}, \mathrm{HCl}, \mathrm{CF}_{3} \mathrm{COOH}\right)$ 转化 为吡喃盐化合物, 苂光增强, 可以作为典型的 off-on 传 感器 $[13]$<smiles>O=c1cc(-c2ccccc2)oc(-c2ccccc2)c1</smiles><smiles>Oc1cc(-c2ccccc2)[o+]c(-c2ccccc2)c1</smiles>

Sheng 和 $\operatorname{Ren}^{[67]}$ 采用计算方法研究了吡喃盐衍生物 对硫化氢的传感机制, 可能的传感机制是酸性条件下水 合阳离子催化了吡喃到噻喃转化. Dahl 等 ${ }^{[68]}$ 合成了芳基 取代的苯并类吡喃盐化合物(Scheme 20), 该类化合物 具有良好的对 $\mathrm{pH}$ 响应光谱性质.
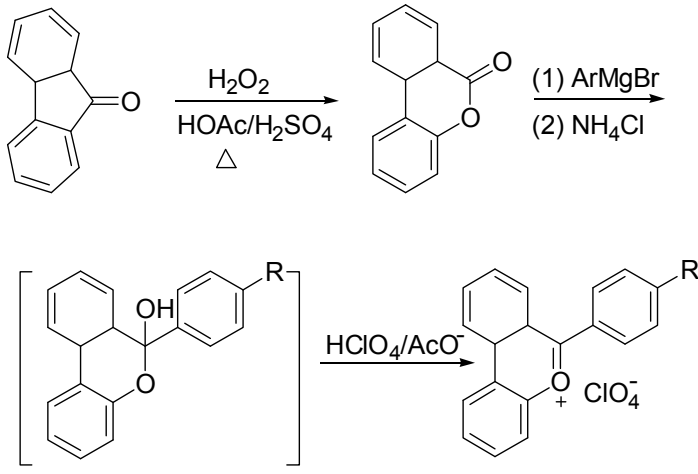

$\mathrm{R}=\mathrm{H}, \mathrm{OCH}_{3}, \mathrm{~N}\left(\mathrm{CH}_{3}\right)_{2}$

31

图式 20 芳基取代苯并类吡喃盐的合成

Scheme 20 Synthesis of aryl-substituted benzopyrylium salt

\section{4 结论和展望}

综上所述，吡喃盐化合物具有特殊的电子结构、光 物理和光化学性质, 吸引了人们的广泛关注. 人们相继 研究出了吡喃酮法、1,5-戊二酮法、查尔酮和苯乙酮衍 生物反应法等多种方法制备吡喃盐化合物，受合成方法 的限制，关于 $\alpha$-活性吡喃盐的研究报道很少，开展 $\alpha$-活 性吡喃盐合成和反应活性研究对合成新材料和开发新 反应具有重要的意义. 同时研究具有不同结构的吡喃盐 的反应活性和性质调控对于开拓新型有机合成反应、开 发新型有机光电材料具有重要科学意义和应用价值. 随 着量子化学计算和合成化学的发展, 性能更丰富的吡喃 盐类化合物将不断涌现，在有机合成中间体、离子液体、 苂光传感、光敏材料、光催化剂等领域会有更为广泛的 应用。

\section{References}

[1] Binnemans, K. Chem. Rev. 2005, 105, 4148.

[2] Saeva, F. D.; Reynolds, G. A.; Kaszczuk, L. J. Am. Chem. Soc. 1982, 104, 3524 .

[3] Pernak, J.; Swierczynska, A.; Kot, M.; Walkiewicz, F.; Maciejewski, H. Tetrahedron Lett, 2011, 52, 4342.

[4] Wang, Y.; He. Q. P.; Li, M. L.; Xu, X. J.; Yin, H. L. Chin. J. Org. Chem. 2008, 28, 718 (in Chinese).

(王勇，赫庆鹏，李满林，许小晶，尹汉东，有机化学，2008，28, 718.)

[5] Dimroth, K.; Greif, N.; Stade, W.; Steuber, F. W. Angew. Chem., Int. Ed. 1967, 6, 711

[6] Abalos, T.; Jimenez, D.; Martinez-Manez, R.; Ros-Lis, J. V.; Royo, S.; Sancenon, F.; Soto, J.;Costero, A. M.; Gil, S.; Parra, M. Tetrahedron Lett. 2009, 50, 3885.

[7] Zhang, J. L.; Zhu, Z. G. Dyes Pigm. 1995, 27, 263.

[8] Gavrilyuk, S.; Polyutov, S.; Jha, P. C.; Rinkevicius, Z.; Agren, H.; Gei'mukhanov, F. J. Phys. Chem. A 2007, 111, 11961

[9] Jayanthi, S. S.; Ramamurthy, P. J. Phys. Chem. A 1998, 102, 511.

[10] Song, X. N.; Mo, L.; Hu, Z. L.; Yang, Z. Y.; Yao, Z. J. Acta Chim. Sinica 2011, 69, 680 (in Chinese). (宋晓男，莫否，胡治隆，杨震宇，姚祝军，化学学报，2011，69, 680.) 
[11] Li, F. Y.; Zheng, J.; Huang, C. H. J. Phys. Chem. B 2000, 104, 5090 .

[12] Scaramuzzo, F. A.; Gonzalez-Campo, A.; Wu, C. C.; Velders, A. H.; Subramaniam, V.; Doddi, G.; Mencarelli, P.; Barteri, M.; Jonkheijm, P.; Huskens, J. Chem. Commun. 2010, 46, 4193.

[13] Zhou, T. L.; Jia, T.; Zhao, S. S.; Guo, J. H.; Zhang, H. Y.; Wang, Y. Cryst. Growth Des. 2012, 12, 179.

[14] Müller, C.; Freixa, Z.; Lutz, M.; Spek, A. L.; Vogt, D.; van Leeuwen, P. W. N. M. Organometallics 2008, 27, 834.

[15] Liebscher, J.; Harumann, H. Z. Chem. 1973, 13, 342.

[16] Balaban, T. S.; Balaban, A. T. Sci. Synth. 2003, 14, 11.

[17] Ning, G. L.; Li, X. C.; Munakata, M.; Gong, W. T.; Maekawa, M.; Kamikawa, T. J. Org. Chem. 2004, 69, 1432.

[18] Ye, J. W.; Zhang, X. D.; Deng, D.; Ning, G. 1.; Liu, T. Q.; Zhuang, M. L.; Yang, L. J.; Gong, W. T.; Lin, Y. RSC Adv. 2013, 3, 8232.

[19] Gong, W. T.; Ning, G. L.; Li, X. C.; Wang, L.; Lin, Y. J. Org. Chem. 2005, 70, 5768 .

[20] Yang, L. J.; Ye, J. W.; Gao, Y.; Deng,D.; Gong, W. T.; Li, Y.; Ning, G. L. Tetrahedron Lett. 2013, 54, 2967.

[21] Xu, T.; Gong, W. T.; Ye, J. W.; Li, Y.; Ning, G. L. Organometallics 2010, 29, 6744.

[22] Li, G.; Gong, W. T.; Ye, J. W.; Li, Y.; Ning, G. L. Synth. Commun. 2012, 42, 480.

[23] Zhang, X. D.; Ye, J. W.; Wang, S. N.; Gong, W. T.; Li, Y.; Ning, G. L. Org. Lett. 2011, 13, 3608 .

[24] Yang, L. J.; Ye, J. W.; Gao, Y.; Deng,D.; Li, Y.; Ning, G. L. Eur. J. Org. Chem. 2014, 3, 515.

[25] Li, G.; Gong, W. T.; Ye, J. W.; Lin, Y.; Ning, G. L. Chin. J. Org. Chem. 2011, 31, 216 (in Chinese). (李刚, 贡卫涛, 叶俊伟, 林源, 宁桂玲, 有机化学, 2011, 31, 216.)

[26] Krivun, S. V.; Buryak, A. I.; Baranov, S. N. Chem. Heterocycl. Compd. 1973, 9, 1191.

[27] Balaban, A. T.; Dinculescu, A.; Dorofeenko, G. N.; Fischer, G. W.; Koblik, A. V.; Mezheritskii, V. V.; Schroth, W. Adv. Heterocycl. Chem. 1982, (Suppl. 2), 184.

[28] Vanallan, J. A.; Reynolds, G. A. J. Org. Chem. 1968, 33, 1102.

[29] Fakis, M.; Polyzos, J; Tsigaridas, G.; Parthenios, J.; Fragos, A.; Giannetas, V.; Persephonis, P.; Mikroyannidis, J. Chem. Phys. Lett. 2000, 323, 111.

[30] Cheng, X. H.; Hoger, S.; Fenske, D. Org. Lett. 2003, 5, 2587.

[31] Krivun S. V.; Shiyan, Z. V.; Dorofeenko, G. N.. Zh. Obshch. Khim. 1964, 34, 167.

[32] Balaban, A. T.; Nenitzesco, C. D. J. Chem. Soc. 1961, 3553.

[33] Balaban, A. T.; Nenitzescu, C. D. Org. Synth. 1964, 44, 98.

[34] Hopf, P. P.; Le Fevre, R. J. W. J. Chem. Soc. 1938, 1989.

[35] Katritzky, A. R.; Vassilatos, S. N.; Alajarin, C. M. Org. Magn. Res. 1983, 21, 587.

[36] van der Velde, N. A.; Korbitz, H. T.; Garner, C. M. Tetrahedron Lett. 2012, 53, 5742.

[37] Tovar, J. D.; Swager, T. M. J. Org. Chem. 1999, 64, 6499.
[38] Shaw, M.J.; Mertz, J. Organometallics 2002, 21, 3434.

[39] Lombard, R. Stephan, J. P. Bull. Soc. Chim. Fr. 1958,1458.

[40] Kuhnert, N.; Clifford, M. N.; Radenac, A.G. Tetrahedron Lett. 2001, 42, 9261.

[41] Bello, A. M.; Kotra, L. P. Tetrahedron Lett. 2003, 44, 9271.

[42] Prasad, N.; Main, L.; Nicholson, B. K.; McAdam, C. J. J. Organomet. Chem. 2010, 695, 1961.

[43] Liebscher, J.; Hartmann, H. Z. Chem. 1973, 13, 132.

[44] Schroth, W.; Fischer, G. W.; Rottmann, J. Chem. Ber. 1969, 102, 1201.

[45] Dorofeenko, G. N.; Mezheritskii, V. V. Khim. Geterotsikl. Soedin. 1970, 2, 217

[46] Simalty, M.; Carretto, J.; Sib, S. Bull. Soc. Chim. Fr. 1970, 11, 3920.

[47] Laine, P.; Bedioui, F.; Ochsenbein, P.; Marvaud, V.; Bonin, M.; Amouyal, E. J. Am. Chem. Soc. 2002, 124, 1364.

[48] Bhowmik, P. K.; Han, H.; Cebe, J. J.; Nedeltchev, I. K.; Kang, S. W.; Kumar, S. Macromolecules 2004, 37, 2688.

[49] Wu, D. Q.; Zhi, L. J.; Bodwell, G. J.; Cui, G. L.; Tsao, N.; Müller, K. Angew. Chem., Int. Ed. 2007, 46, 5417.

[50] Uncuta, C.; Caproiu, M. T.; Campeanu, V.; Petride, A.; Danila, M. G.; Plaveti, M.; Balaban, A. T. Tetrahedron 1998, 54, 9747.

[51] Mouradzadegun, A.; Dianat, S. J. Heterocycl. Chem. 2009, 46, 778.

[52] Tolmachev, A. I.; Koslov, E. S. Zh. Obshch. Khim. 1967, 37, 1922.

[53] Markl, G.; Lieb, F.; Merz, A. Angew. Chem., Int. Ed. 1967, 6, 458.

[54] Krivun, S. V.; Voziyano, O. F.; Baranov, S. N. Zh. Obshch. Khim. 1972, 42, 58

[55] Kamata, M.; Kaneko, J.; Hagiwara, J.; Akaba, R. Tetrahedron Lett. 2004, 45, 7423.

[56] Miranda, M. A.; Garcia, H. Chem. Rev. 1994, 94, 1063.

[57] Branchi, B.; Bietti, M.; Ercolani, G.; Izquierdo, M. A.; Miranda, M. A.; Stella, L. J. Org. Chem. 2004, 69, 8874.

[58] Liang, S.; Zhu, J. K.; Hurst, J. K. Langmuir. 2012, 28, 12171.

[59] Zlotin, S. G.; Makhova, N. N. Mendeleev Commun. 2010, 20, 63.

[60] Saeva, F. D.; Reynolds, G. A.; Kaszczuk, L. J. Am. Chem. Soc. 1982, 104, 3524.

[61] Gionis, V.; Fugnitto, R.; Strzelecka, H.; Le Barny, P. Mol. Cryst. Liq. Cryst. 1983, 95, 351.

[62] Veber, M.; Berruyer, G. Liq. Cryst. 2000, 27, 671.

[63] Pernak, J.; Swierczynska, A.; Kot, M.; Walkiewicz, F.; Maciejewski, H. Tetrahedron Lett. 2011, 52, 4342.

[64] Chen, Y.; Wu, S. K. Chem. J. Chin. Univ. 1996, 17, 1622 (in Chinese). (陈懿, 吴世康, 高等学校化学学报, 1996, 17, 1622.)

[65] Haucke, G.; Czerney, P.; Cebulla, F. Ber. Bunsen-Ges. Phys.Chem. 1992, 96, 880 .

[66] Azab, H. A.; El-Korashy, S. A.; Anwar, Z. M.; Khairy, G. M.; Duerkop, A. J. Photochem. Photobiol., A 2012, 243, 41.

[67] Sheng, Y. H.; Ren, Y. J. Phys. Chem. A 2012, $116,5420$.

[68] Prust, E. E.; Carlson, E. J.; Dahl, B. J. Tetrahedron Lett. 2012, 53, 6433. 